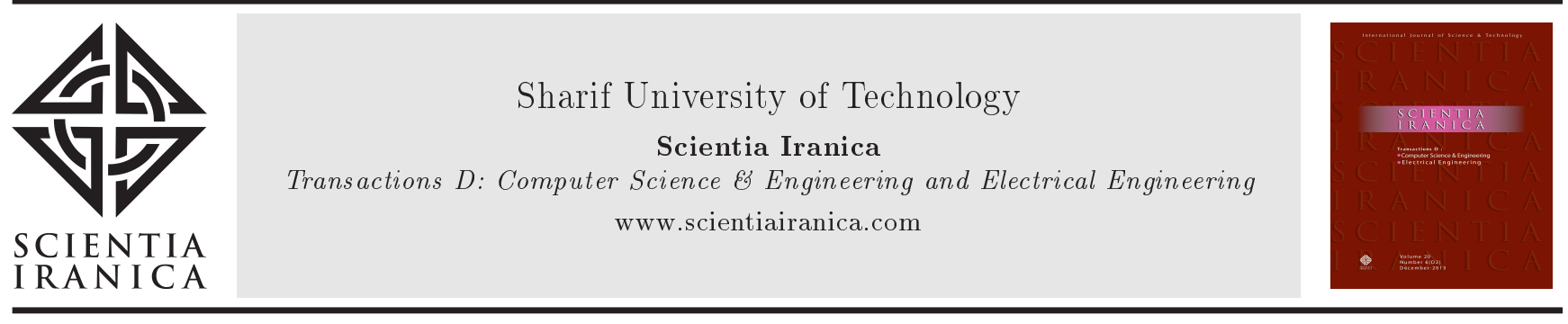

\title{
A new adequacy evaluation method for transmission expansion planning problems
}

\author{
M.R. Habibi ${ }^{a, *}$, M. Rashidinejad ${ }^{b}$ and A. Abdollahi ${ }^{b}$ \\ a. Department of Electrical Engineering and Computer Science, Kerman Graduate University of Technology, Kerman, Iran. \\ b. Department of Electrical Engineering, Shahid Bahonar University of Kerman, Kerman, Iran.
}

Received 2 March 2015; received in revised form 27 September 2015; accepted 26 December 2015

\section{KEYWORDS \\ DC operation model; \\ Superposition \\ principle; \\ Transmission \\ expansion planning; \\ Transmission system \\ adequacy evaluation.}

\begin{abstract}
Transmission Expansion Planning (TEP) problem refers to proposing the optimal number and location of new transmission lines in order to satisfy operation conditions with less investment costs. Adequacy evaluation of trial solutions is essential to solve TEP problem. This evaluation procedure may require a considerable computational effort; therefore, improvement of Adequacy Evaluation Methods (AEMs) is a key to achieve more efficient TEP solution algorithms. It is a common practice to employ a Linear Programming (LP) called "DC operation model" to perform this evaluation, especially in cases that generation rescheduling is considered in TEP studies. In this paper, an AEM is proposed as an alternative with less computational effort to perform adequacy evaluation. In fact, the proposed AEM is applicable in TEP problems considering generation rescheduling. The study is based on DC TEP model where electrical aspect of the problem is modeled based on DC power flow equations. To examine the performance of the proposed AEM, this evaluation method is employed in a meta-heuristic TEP solution algorithm. Such a combinatorial algorithm is then applied to different standard test systems as well as practical cases. The simulation results show a promising improvement caused by employment of the proposed AEM.

(C) 2016 Sharif University of Technology. All rights reserved.
\end{abstract}

\section{Introduction}

Transmission Expansion Planning (TEP) is one of the most important parts of the future development in power systems. TEP problems will offer an optimal plan for transmission lines with less investment costs in a prescribed planning horizon. An optimal expansion of transmission systems should be capable of facilitating different load patterns as well as future generations $[1,2]$. The desired solution of TEP problems is an adequate network with minimum construction costs [3].

Various TEP models are presented in the lit-

*. Corresponding author.

E-mail addresses: m.habibi@student.kgut.ac.ir (M.R.

Habibi); mrashidi@uk.ac.ir (M. Rashidinejad);

a.abdollahi@uk.ac.ir (A. Abdollahi) erature, like reliability-constrained TEP, TEP with FACTS devices, and TEP associated with reactive power planning [4-6]. The DC model can be considered as a basic model of this problem. Mathematically, DC TEP is a mixed integer, non-linear, and non-convex optimization problem. In literature, optimization methods for solving DC TEP are categorized into three groups:

(a) Classical algorithms;

(b) Heuristic algorithms (mostly constructive heuristics);

(c) Meta-heuristics.

Most heuristic and meta-heuristic methods presented in the field of TEP have a particular stage to perform adequacy evaluations [7-11]. Such evaluation 
is performed repeatedly and may totally require a considerable computational effort. Therefore, employment of more efficient AEMs will reduce the total computational effort for TEP solution algorithms [12].

"DC operation model" is a common AEM for DC TEP model, where it calculates the minimum required load shedding satisfying network power flow limits. In fact, it employs an LP to perform such calculations, in which the system is adequate if and only if the required load shedding tends to zero [13].

To remove an overload in a transmission system, either "load curtailment" or "generation rescheduling" can be performed, alternatively. Therefore, DC operation model has been presented for two different forms of DC TEP problems: either with generation rescheduling or without generation rescheduling. Solving a DC TEP problem with generation rescheduling considers operational constraints, while a DC TEP without generation rescheduling is based upon a fixed predefined generation pattern $[12,14]$.

In our previous work [12], an efficient AEM was presented for DC TEP without generation rescheduling, where it was associated with DC power flow and graph theory. Since it is not applicable for the case with generation rescheduling, it should be modified in order to handle generation rescheduling.

In this paper, the AEM presented in [12] is modified while generation rescheduling is taken into consideration. This work is mainly based on graph theory, DC power flow calculations, and superposition principle. To compare its performance with that of DC operation model, AEM in employed in the scatter search algorithm. The computational effort of such combined model in comparison with the work in [13] is reported to illustrate efficiency of the proposed algorithm

This paper is organized as follows: DC operation model is presented in Section 2; the proposed AEM is discussed Section 3; while a brief description of its usage in a sample meta-heuristic procedure is provided in Section 4; simulation studies and the analysis of results are provided in Section 5; finally, concluding remarks are driven in Section 6 and some necessary calculations are provided in the Appendix.

\section{DC operation model}

A DC operation model is a common AEM in DC TEP studies. Considering generation rescheduling, the problem is formulated as follows:

$$
\min w=\sum r_{k}
$$

Subject to:

$$
S f+g+d=r,
$$

$$
\begin{aligned}
& f_{i j}-\gamma_{i j} n_{i j}^{1}\left(\theta_{i}-\theta_{j}\right)=0, \\
& \left|f_{i j}\right| \leq n_{i j}^{1} \bar{f}_{i j}, \\
& 0 \leq g \leq \bar{g}, \\
& 0 \leq r \leq d, \\
& (i, j) \in \Omega .
\end{aligned}
$$

In this formulation, $n_{i j}^{1}$ represents the number of circuits in the right of way $i-j$ and $n_{i j}^{1}$ is a given input to this model. The main output of this model is the variable $w$ which represents the minimum load shedding required to eliminate power flow violations. The network is considered to be adequate if and only if $w=0$. In the above LP model, the objective is to minimize the total applied load curtailment to the power system. Eq. (1a) refers to conversation of active power in each bus (i.e., known as power KCL). Eq. (1b) denotes the relation between network power flows and phase angle at buses. This equation is also known as power KVL. Line flow limits, power generation limits, and load curtailment limits are presented in Eqs. (1c), (1d), and (1c), respectively. It can be observed that interconnectivity of the network is not considered in the mathematical model.

\section{The proposed AEM}

In this section, a new AEM is proposed for evaluating network adequacy in which an interconnected network is adequate if a generation pattern satisfies the following requirements, simultaneously:

- Total generating power must be equal to demand (power balance constraint);

- The generating output power must be satisfied (generation limit constraint);

- The network flow for such generation pattern must satisfy line power flow limits (network flow limit constraint).

The adequacy of the system is equivalent to network overload removal for such a feasible generation pattern. In addition, a non-interconnected transmission system is adequate if all of its sub-topologies are adequate. Figure 1 illustrates the framework for evaluating the adequacy of transmission network considering generation rescheduling with the following steps:

Step 1: Determine sub-topologies in the current network;

Step 2: If any of the sub-topologies has a total generation capacity less than its demand, then load curtailment must be necessary and the system is not adequate. Hence, the process will be terminated; otherwise, go to Step 3; 


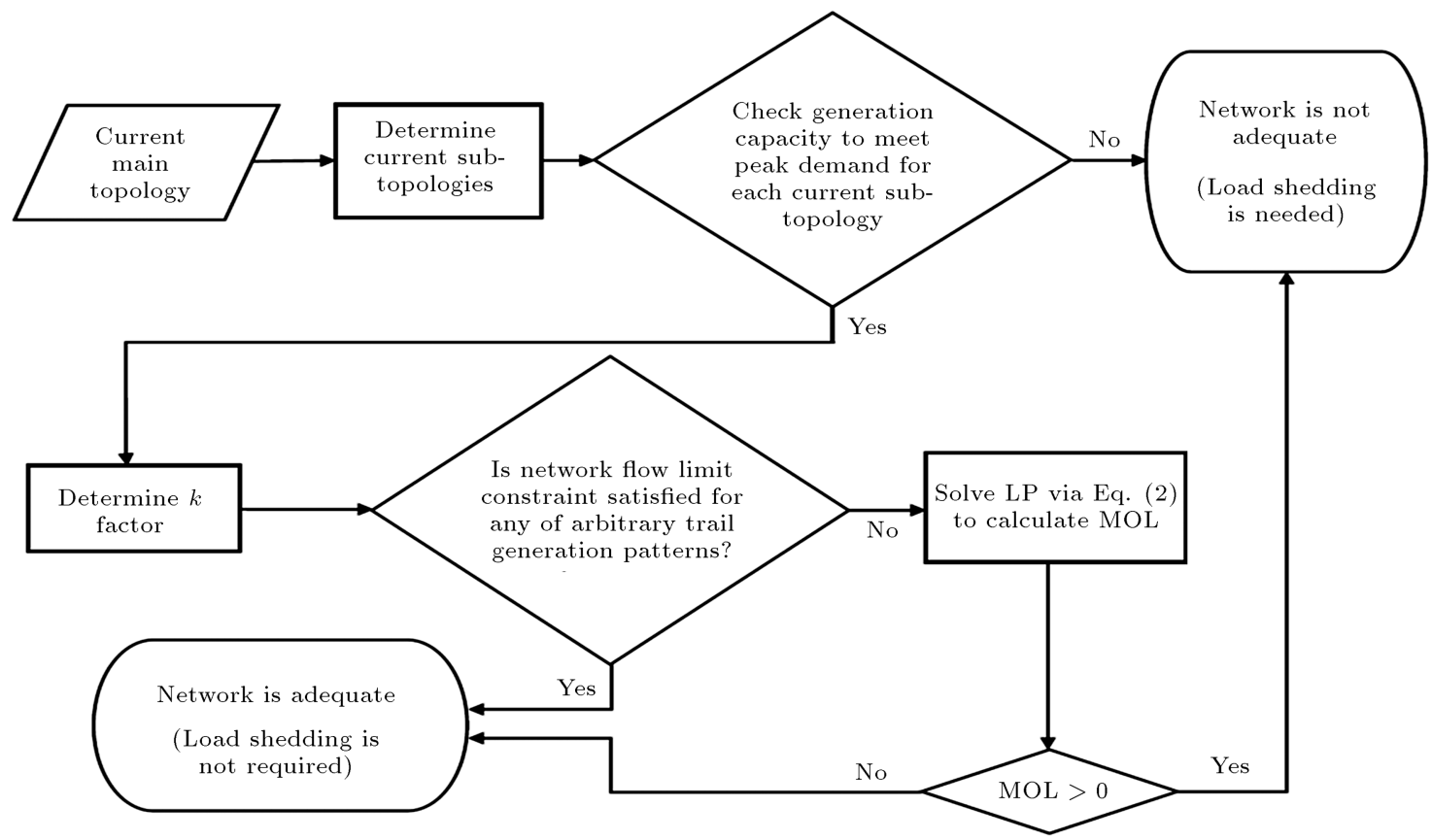

Figure 1. The proposed algorithm flow diagram.

Step 3: For each sub-topology, do Steps 4 and 5 to evaluate its adequacy evaluation. The network is considered to be adequate if and only if all subtopologies are determined to be adequate;

Step 4: In order to provide a fast method to calculate power flow, build up the following linear equation based upon the superposition principle $[13,15]$ :

$$
f_{i j}=\sum_{g \in \Psi_{\mathrm{part}}} k_{g, i j} P_{g} .
$$

Calculation of $k_{g, i j}$ factors is described in the Appendix. Consider some arbitrary trial generation patterns satisfying both the "power balance constraint" and "Generation limit constraint". Calculate network power flows using Eq. (2). Then, check "network flow limit constraint". If these three constraints are satisfied for any of the generating patterns, the current sub-topology is adequate; then, return to Step 3 and check the next sub-topology.

Step 5: To find a feasible generating pattern that satisfies system requirements, the LP in Eq. (3) is employed. Maximum overload (MOL) refers to the maximum value among overloads through the whole network. This LP tries to find the minimum value of MOL that can be obtained by generation rescheduling.

$\min$ MOL.

Subject to:

$\sum_{g \in \Psi_{\text {part }}} P_{g}=D_{\text {part }}, \quad \forall$ part $=1, \cdots, n_{\text {part }}$,

$$
\begin{aligned}
& 0 \leq P_{g} \leq P_{g}^{\max }, \quad \forall \quad g \quad \in \Psi_{\text {part }}, \\
& \left|\sum_{g=1}^{n_{\text {gen }}} k_{g, i j} P_{g}\right| \leq n_{i j}^{1} \bar{f}_{i j}+\mathrm{MOL}, \quad \forall \quad(i, j) \in \Omega, \\
& \text { MOL } \geq 0 .
\end{aligned}
$$

If MOL is zero, then the current sub-topology is adequate and return to Step 3; otherwise, the whole network is not adequate.

The advantage of using LP (Eq. (3)) rather than DC operation model is that it has less number of variables and less number constraints, which will be demonstrated by numerical studies in Section 5 . Therefore, the proposed AEM is more efficient than DC operation model.

\section{AEM and a meta-heuristic algorithm}

To assess the performance of the proposed methodology, AEM should be considered as a sub-procedure in a heuristic or meta-heuristic algorithm. In this work, the DC operation model in [13] is replaced by the proposed AEM, where it is called "Altered Scatter Search Algorithm" (ASSA). In the next section, the improvement caused by this modification is studied as a measure for the proposed AEM performance.

\section{Case studies and analysis of results}

In this section, to study the impacts of the proposed AEM, the computational effort of ASSA is determined. 
The proposed algorithm is applied to Garver, 24-bus IEEE, 46-bus Brazilian systems, and also the 54-bus Iran Southeast Network. The simulation studies are based on a DC-TEP considering generation rescheduling. It is remarkable that applying the proposed AEM merely reduces the computational effort without any effect on the final optimal solution.

\subsection{Garver's system}

This system includes six transmission lines and six buses with 760 MW demand for the base topology which is shown in Figure 2. The solid lines represent the existing circuits in the base case topology and the dotted lines represent the candidate possible right of ways.

The number of candidate lines is 15 circuits. The system data can be found in [16]. The optimal solutions, obtained by applying both algorithms to Garver system with generation rescheduling, are presented in Table 1.

As it was expected, employing the proposed AEM does not lead the algorithm to a different optimal solution. In Table 2, the number of LPs solved to

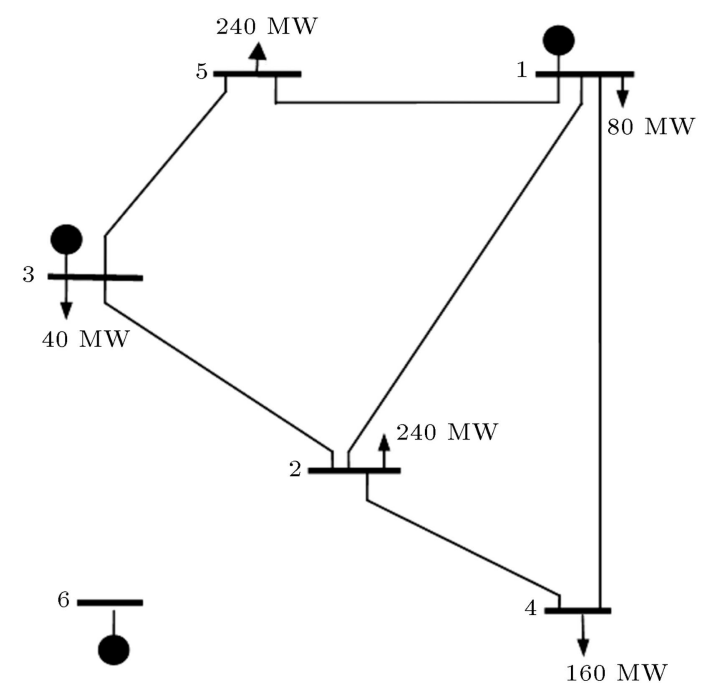

Figure 2. Initial network of Garver system.

Table 1. The obtained optimal solutions via both algorithms for Garver system.

\begin{tabular}{ccc}
\hline Method & Added circuits & $\begin{array}{c}\text { Total investment } \\
\text { cost }(\mathbf{M} \$)\end{array}$ \\
\hline$[13]$ & $n_{3-5}=1, n_{4-6}=3$ & 110 \\
ASSA & $n_{3-5}=1, n_{4-6}=3$ & 110 \\
\hline
\end{tabular}

Table 2. Number of solved LPs in both algorithms for Garver system.

\begin{tabular}{cc}
\hline Method & Number of LPs \\
\hline$[13]$ & $209-300$ \\
ASSA & $50-64$ \\
\hline
\end{tabular}

obtain the optimal solution is shown. It can be noticed that the presented ASSA shows a better performance than [13], because it executes fewer LPs to find the optimal solution for the tested system.

\subsection{IEEE 24-bus system}

IEEE 24-bus system consists of 24 buses and 41 right of ways for the addition of new circuits with $8550 \mathrm{MW}$ demand for the base topology, which is shown in Figuer 3. The data is available in [17]. By applying both algorithms to this system considering generation rescheduling, the obtained results are presented in Table 3.

The numbers of solved LPs to obtain the optimal solution for both algorithms are compared in Table 4 . As it can be noticed, the ASSA shows a better

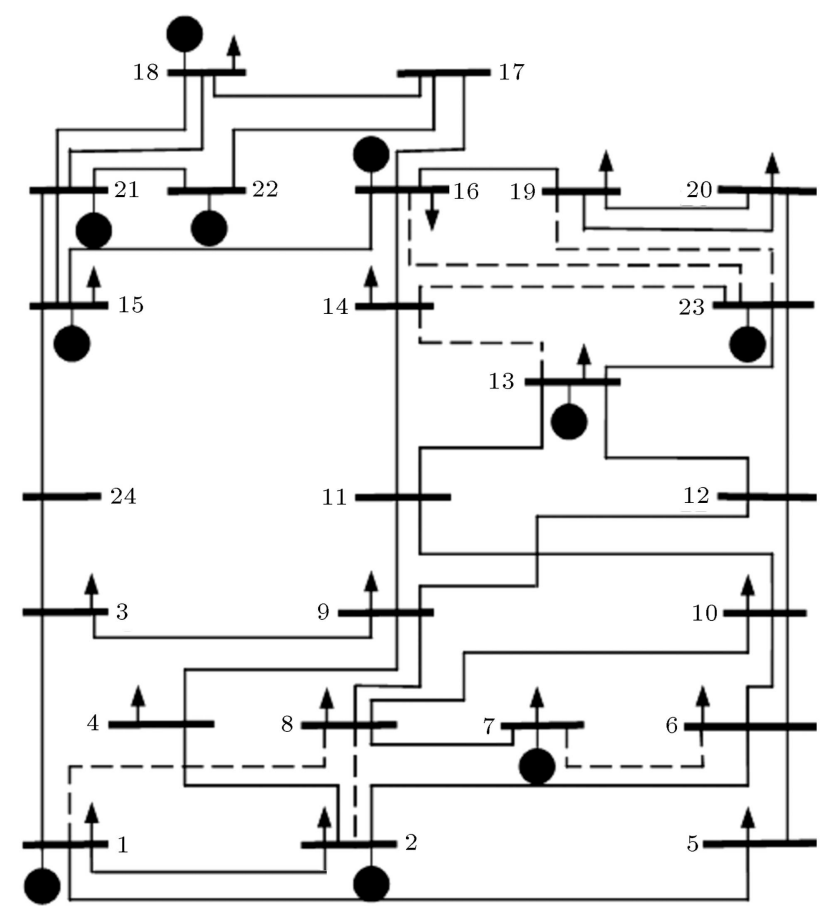

Figure 3. Initial network of IEEE 24-bus system.

Table 3. The obtained optimal solutions via both algorithms for IEEE 24-bus system.

\begin{tabular}{ccc}
\hline Method & Added circuits & $\begin{array}{c}\text { Total investment } \\
\text { cost }(\mathbf{M} \$)\end{array}$ \\
\hline$[13]$ & $n_{6-10}=1, n_{7-8}=2$, & 152 \\
& $n_{10-12}=1, n_{14-16}=1$ & \\
ASSA & $n_{6-10}=1, n_{7-8}=2$, & 152 \\
& $n_{10-12}=1, n_{14-16}=1$ & \\
\hline
\end{tabular}

Table 4. Number of solved LPs in both algorithms for IEEE 24-bus system.

\begin{tabular}{cc}
\hline Method & Number of LPs \\
\hline$[13]$ & $310-610$ \\
ASSA & $119-165$ \\
\hline
\end{tabular}


performance, because it executes fewer LPs to find the optimal solution for the tested system.

\subsection{Southern Brazilian system of 46 buses}

Southern Brazilian System has 46 buses, 79 right of ways for the addition of new circuits, and $6880 \mathrm{MW}$ of demand. The system data is available in [18]. The base topology of this system is shown in Figure 4. There is no limit for circuit additions in each right of way. The proposed algorithm offered the results shown in Table 5.

In Table 6, the number of solved LPs to obtain the optimal solution is shown. The ASSA shows a better performance than [13], because it executes fewer LPs to find the optimal solution for the tested system.

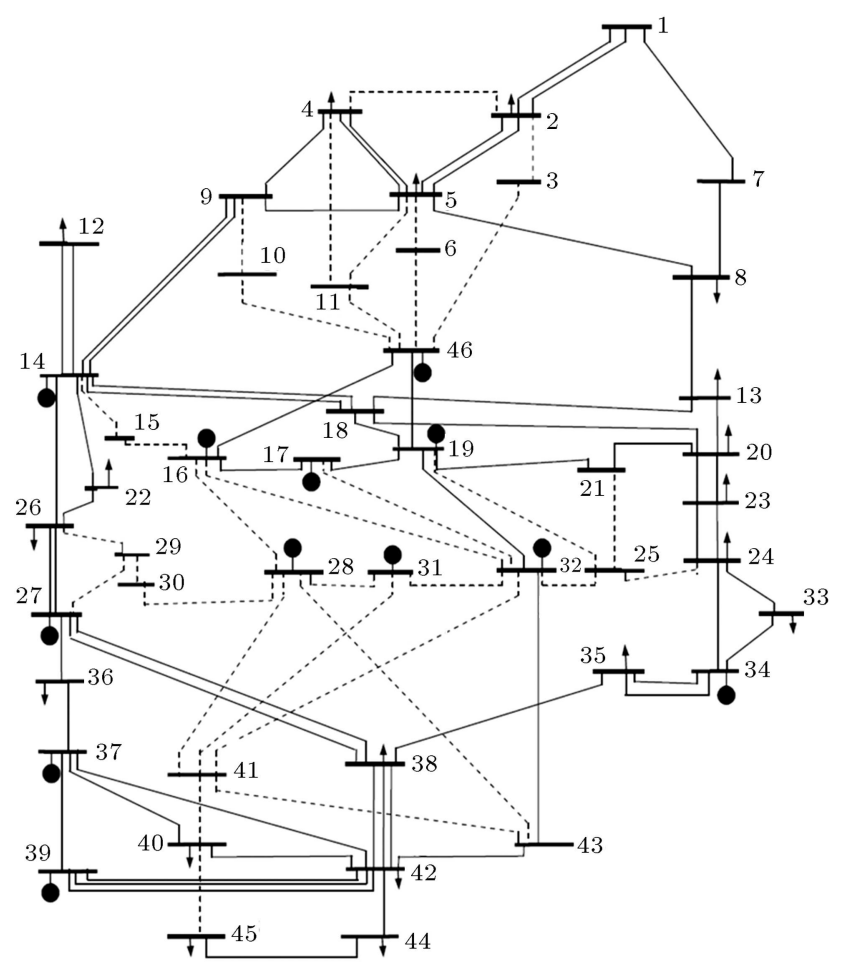

Figure 4. The base topology of southern Brazilian system of 46 buses.

Table 5. The obtained optimal solutions via both algorithms for 46-bus system.

\begin{tabular}{ccc}
\hline Method & Added circuits & $\begin{array}{c}\text { Total investment } \\
\text { cost (M\$) }\end{array}$ \\
\hline \multirow{3}{*}{$n_{2-5}=1, n_{5-6}=2}$, & \\
& $n_{13-20}=1, n_{20-21}=2$, & 72.87 \\
& $n_{20-23}=1, n_{42-43}=1$, & \\
& $n_{46-6}=1$. \\
& \\
ASSA $\quad n_{13-5}=1, n_{5-6}=2$, & \\
& $n_{20-23}=1, n_{42-43}=1$, & \\
& & \\
& & \\
&
\end{tabular}

Table 6. Number of solved LPs in both algorithms for 46-bus system.

\begin{tabular}{cc}
\hline Method & Number of LPs \\
\hline$[13]$ & $15219-53861$ \\
ASSA & $1742-13179$ \\
\hline
\end{tabular}

\subsection{Southeast Network of Iran (SNI) of 51 buses}

In this section, the proposed method is applied to the southeast network of Iran. Southeast network of Iran is a part of the interconnected electric power network in Iran, which is divided into 4 regions (provinces): Kerman, Yazd, Hormozgan, and Sistan-Baluchestan. Southeast network of Iran procures the electric power for an area of about $381787 \mathrm{~km}^{2}$. Main tie lines between southeast network and other regions are: four $(2 * 400 \mathrm{KV} \& 2 * 230 \mathrm{KV})$ lines connected to Esfahan and three lines $(2 * 400 \mathrm{KV} \& 1 * 230 \mathrm{KV})$ connected to Fars. The total length of $230 \mathrm{kV}$ lines is about $2372 \mathrm{~km}$ and the total length of $400 \mathrm{kv}$ lines is about $4635 \mathrm{~km}$.

This system has 51 buses and 89 right of ways for the addition of new circuits and a total demand of $10268 \mathrm{MW}$. The base year topology is 2010 and the expansion is targeted for year 2016. Single line diagram of southeast network of Iran is illustrated in Figure 5 .

By applying both algorithms to southeast network of Iran, the results shown in Table 7 have been obtained.

In Table 8, the number of solved LPs to obtain the optimal solution time for both algorithms is shown. The ASSA shows a better performance than [13], because it executes fewer LPs to find the optimal solution for the tested system.

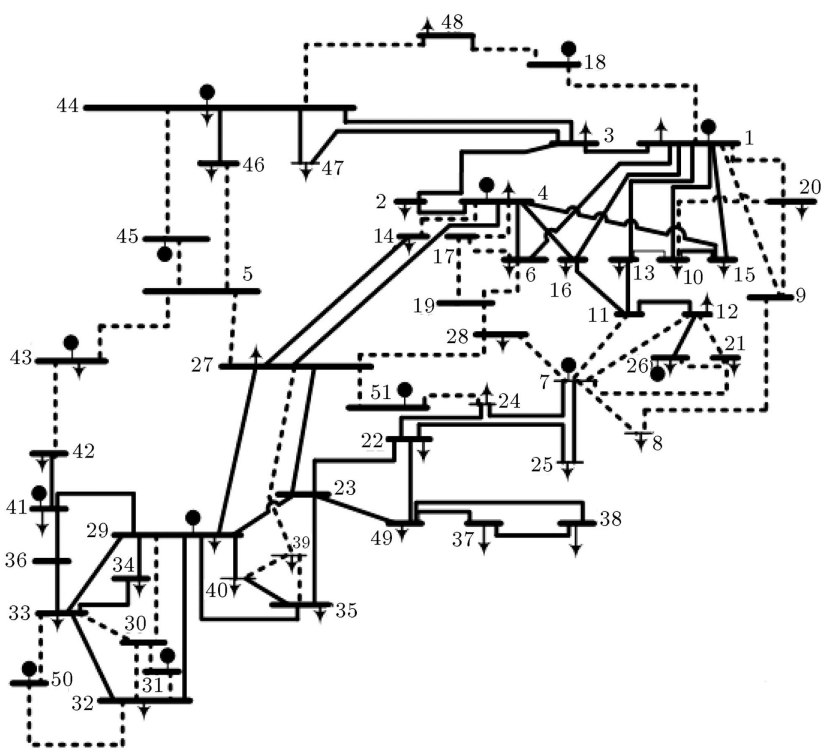

Figure 5. Single diagram of Iran Southeast Network. 
Table 7. The obtained optimal solutions via different methods for SNI.

\begin{tabular}{|c|c|c|}
\hline Method & Added circuits & $\begin{array}{c}\text { Total } \\
\text { investment } \\
\text { cost }(\mathrm{M} \$)\end{array}$ \\
\hline [13] & $\begin{array}{c}n_{7,21}=1, n_{7,28}=1, \\
n_{4,14}=1, n_{7,8}=1, \\
n_{10,20}=1, n_{29,35}=1, \\
n_{35,39}=1, n_{24,51}=2, \\
n_{33,50}=2, n_{27,28}=1, \\
n_{42,43}=1, n_{27,51}=1 .\end{array}$ & 64.27 \\
\hline ASSA & $\begin{array}{c}n_{7,21}=1, n_{7,28}=1, \\
n_{4,14}=1, n_{7,8}=1, \\
n_{10,20}=1, n_{29,35}=1, \\
n_{35,39}=1, n_{24,51}=2, \\
n_{33,50}=2, n_{27,28}=1, \\
n_{42,43}=1, n_{27,51}=1 .\end{array}$ & 64.27 \\
\hline
\end{tabular}

Table 8. Number of solved LPs in both methods for SNI.

\begin{tabular}{cc}
\hline Method & Number of LPs \\
\hline$[13]$ & $753-2052$ \\
ASSA & $253-694$ \\
\hline
\end{tabular}

\section{Concluding remarks}

This paper presents an efficient Adequacy Evaluation Method (AEM) for DC TEP considering generation rescheduling. This AEM evaluates adequacy of a power system based on graph theory, DC power flow calculations, and the superposition principle.

The proposed evaluation method has been integrated in a meta-heuristic procedure [13] and applied to test systems including Garver, IEEE 24-bus, the southern Brazilian system of 46 buses, and also 54bus Southeast Network of Iran. The obtained results for these test systems show that integration of the proposed AEM can lead to a significant computational performance in comparison with the case that DC operation model is employed to evaluate adequacy of solutions. Also, because of the equivalency of the proposed AEM with the DC operation model, integration of this AEM does not lead the meta-heuristic algorithm to a different solution.

Modification of the proposed AEM for more complete models of TEP problems can be suggested for future works. The proposed AEM is based on a static model of TEP which considers the one-time horizon for transmission planning, so it is valuable to expand the AEM to a multi-period planning model. Considering uncertainties in the planning and development of this AEM for AC model of TEP is also suggested to improve this work.

\author{
Nomenclature \\ $d \quad$ The vector with elements $d_{k}$ (demand \\ at bus $k$ ) \\ $D_{\text {part }} \quad$ Total demand in a part (sub-topology) \\ of network \\ $f \quad$ A vector with elements $f_{i j}$ \\ $f_{i j} \quad$ Active power flow through line $i-j$ \\ $\bar{f}_{i j} \quad$ Maximum active power flow limit of \\ line $i-j$ \\ $g$ \\ $g_{k} \quad$ Generation at bus $k$ \\ $\bar{g} \quad$ The vector of the maximum generation \\ limits of generators \\ $n_{\text {part }} \quad$ Number of sub-topologies in the \\ network \\ $n_{\text {gen }} \quad$ The number of generators in the \\ current sub-topology \\ $n_{i j}^{l} \quad$ The number of lines in the right of way \\ $i-j$ \\ $P_{g} \quad$ The generation power of the $g_{t h}$ \\ generator \\ $P_{g}^{\max } \quad$ The maximum generation capacity of \\ the $g_{t h}$ generator \\ $r$ \\ A vector with elements $r_{k}$ \\ $r_{k} \quad$ Load curtailment in load bus $k$ \\ $S \quad$ Transpose branch-node incidence \\ matrix \\ $w \quad$ The minimum required load \\ curtailment of the power system \\ $\Omega \quad$ The set of all possible right of ways \\ $\gamma_{i j} \quad$ Susceptance of line $i-j$ \\ $\theta_{i} \quad$ Phase angle at bus $i$ \\ $\psi_{\text {part }} \quad$ The set of all generators in a part \\ (sub-topology) of network
}

\section{References}

1. Escobar, A.H., Gallego, R.A. and Romero, R. "Multistage and coordinated planning of the expansion of transmission systems", Power Systems, IEEE Transactions on, 19, pp. 735-744 (2004).

2. Rahmani, M., Rashidinejad, M., Gharaveisi, A. and Mohammadian, M. "Transmission network expansion planning using AC model via real GA", International Conference on Power Control \& Optimization, Thailand (2008).

3. Murugan, P. "Modified particle swarm optimisation with a novel initialisation for finding optimal solution to the transmission expansion planning problem", Generation, Transmission \& Distribution, IET, 6, pp. 1132-1142 (2012). 
4. Molina, J.D., Contreras, J. and Rudnick, H. "A riskconstrained project portfolio in centralized transmission expansion planning", Systems Journal, IEEE, 99, pp. 1-9 (2014).

5. Blanco, G., Olsina, F., Garces, F. and Rehtanz, C. "Real option valuation of FACTS investments based on the least square Monte Carlo method", Power Systems, IEEE Transactions on, 26, pp. 1389-1398 (2011).

6. Hui, Z., Heydt, G.T., Vittal, V. and Quintero, J. "An improved network model for transmission expansion planning considering reactive power and network losses", Power Systems, IEEE Transactions on, 28, pp. 3471-3479 (2013).

7. Lee, C., Ng, S.K., Zhong, J. and Wu, F.F. "Transmission expansion planning from past to future", 2006 IEEE PES Power Systems Conference and Exposition (PSCE), pp. 257-265 (2006).

8. Verma, A., Panigrahi, B. and Bijwe, P. "Harmony search algorithm for transmission network expansion planning", Generation, Transmission \& Distribution, IET, 4, pp. 663-673 (2010).

9. Cortes-Carmona, M., Palma-Behnke, R. and Moya, O. "Transmission network expansion planning by a hybrid simulated annealing algorithm", 15th International Conference on Intelligent System Applications to Power Systems (ISA P2009.), pp. 1-7 (2009).

10. Sum-Im, T., Taylor, G., Irving, M. and Song, Y. "Differential evolution algorithm for static and multistage transmission expansion planning", Generation, Transmission \& Distribution, IET, 3, pp. 365-384 (2009).

11. Eghbal, M., Saha, T.K. and Hasan, K.N. "Transmission expansion planning by meta-heuristic techniques: A comparison of shuffled frog leaping algorithm, PSO and GA", in Power and Energy Society General Meeting, pp. 1-8 (2011).

12. Habibi, M.R., Rashidinejad, M., Zeinaddini-Meymand, M. and Fadainejad, R. "An efficient scatter search algorithm to solve transmission expansion planning problem using a new load shedding index", International Transactions on Electrical Energy Systems, 24(2), pp. 153-165 (2013).

13. Meymand, M.Z., Rashidinejad, M., Khorasani, H., Rahmani, M. and Mahmoudabadi, A. "An implementation of modified scatter search algorithm to transmission expansion planning", Turkish Journal of Electrical Engineering and Computer Sciences, 20, p. 1206 (2012).

14. Villasana, R., Garver, L. and Salon, S. "Transmission network planning using linear programming", Power Apparatus and Systems, IEEE Transactions on, 2, pp. 349-356 (1985).

15. Seifi, H. and Sepasian, M.S., Electric Power System Planning, Springer Science \& Business Media (2011).

16. Romero, R., Monticelli, A., Garcia, A. and Haffner, S. "Test systems and mathematical models for transmission network expansion planning", Generation, Trans- mission and Distribution, IEE Proceedings, 149, pp. 27-36 (2002).

17. Romero, R. and Monticelli, A. "A hierarchical decomposition approach for transmission network expansion planning", Power Systems, IEEE Transactions on, $\mathbf{9}$, pp. 373-380 (1994).

18. Romero, R., Rocha, C., Mantovani, J.R.S. and Sanchez, I.G. "Constructive heuristic algorithm for the DC model in network transmission expansion planning", Generation, Transmission and Distribution, IEE Proceedings, 152, pp. 277-282 (2005).

\section{Appendix}

\section{Calculation of $K$ factors}

The procedure of calculating $k_{g, i j}$ factors is proposed as follows:

Step 1: Consider $n_{\text {gen }}$ linear-independent generation patterns. Present the considered linear independent generation patterns by the following matrix:

$$
P_{n_{\text {gen }}} \times n_{\text {gen }}=\left[\begin{array}{ccc}
P_{1,1} & \cdots & P_{1, n_{\text {gen }}} \\
\vdots & \ddots & \vdots \\
P_{n_{\text {gen }, 1}} & \cdots & P_{n_{\text {gen }}, n_{\text {gen }}}
\end{array}\right]
$$

where, $P_{m, g}$ denotes generating output power of the $g_{t h}$ generator in the generation pattern $m_{t h}$. Any row of this matrix shows a generation pattern;

Step 2: Perform a DCPF calculation for any of these generation patterns;

Step 3: build up the following matrix equation:

$$
F=P \times K
$$

where the matrices $F$ and $K$ are defined as:

$$
\begin{aligned}
& F_{n_{\text {gen }} \times n_{\text {line }}}=\left[f_{m, l}\right], \\
& K_{n_{\text {gen }} \times n_{\text {line }}}=\left[k_{g, l}\right],
\end{aligned}
$$

where, $n_{\text {line }}$ is the number of lines and $f_{m, l}$ is the flow through the line $l_{t h}$ in the generation pattern $m_{t h}$. The desired parameter $k_{g, l}$ represents the participation factor of the $g_{t h}$ generator output in the $l_{t h}$ line flow;

Step 4: Obtain matrix $K$ as follows:

$$
K=P^{-1} F \text {. }
$$

In order to simplify calculations, generation patterns can be considered as follows:

$$
P_{m, g}= \begin{cases}D, & m=g \\ D, & m \neq g\end{cases}
$$


Then, matrix $P$ is considered as follows:

$$
P=D I_{n_{\text {gen }} \times n_{\text {gen }}}
$$

where $I$ refers to identity matrix. Therefore:

$$
P^{-1}=\frac{1}{D} I_{n_{\text {gen }} \times n_{\text {gen }}} .
$$

Considering this generation pattern, obtain matrix $K$ as follows:

$$
K=\frac{1}{D} F \text {. }
$$

\section{Biographies}

Mohammad Reza Habibi was born in Baft, Iran, in 1988. He received his MSc degree in Electrical Engineering from Kerman Graduate University of Technology (KGUT) in Kerman, Iran, in 2011 and is now working for $\mathrm{PhD}$ degree in Electrical Engineering Department at KGUT. His research interests include power system planning, power system optimization, and power system operation.
Masoud Rashidinejad received the BSc degree in Electrical Engineering and the MSc degree in Systems Engineering from Isfahan University of Technology, Isfahan, Iran, and the PhD degree in Electrical Engineering from Brunel University, London, U.K., in 2000. He is currently a Professor in the Department of Electrical Engineering, Shahid Bahonar University of Kerman, Kerman, Iran. His current research interests include power system optimization, power system planning, electricity restructuring, and energy management.

Amir Abdollahi received the BSc degree from Shahid Bahonar University of Kerman, Kerman, Iran, in 2007, the MSc degree from Sharif University of Technology, Tehran, Iran, in 2009, and the PhD degree from Tarbiat Modares University, Tehran, Iran, in 2012, all in Electrical Engineering. He is currently an Assistant Professor in the Department of Electrical Engineering, Shahid Bahonar University of Kerman, Iran. His research interests include demand-side management, planning, reliability, and economics in smart electricity grids. 